\title{
Spatial Correlation of Pathology and Perfusion Changes within the Cortex and White Matter in Multiple Sclerosis
}

\author{
(D)A.D. Mulholland, (D) R. Vitorino, DS.-P. Hojjat, (D)A.Y. Ma, DL. Zhang, (DL. Lee, (D)T.J. Carroll, DC.G. Cantrell, (DC.R. Figley, and \\ (1D.I. Aviv

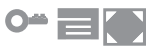

\begin{abstract}
BACKGROUND AND PURPOSE: The spatial correlation between WM and cortical GM disease in multiple sclerosis is controversial and has not been previously assessed with perfusion MR imaging. We sought to determine the nature of association between lobar WM, cortical $\mathrm{GM}$, volume and perfusion.
\end{abstract}

MATERIALS AND METHODS: Nineteen individuals with secondary-progressive multiple sclerosis, 19 with relapsing-remitting multiple sclerosis, and 19 age-matched healthy controls were recruited. Quantitative MR perfusion imaging was used to derive CBF, CBV, and MTT within cortical GM, WM, and T2-hyperintense lesions. A 2-step multivariate linear regression (corrected for age, disease duration, and Expanded Disability Status Scale) was used to assess correlations between perfusion and volume measures in global and lobar normalappearing WM, cortical GM, and T2-hyperintense lesions. The Bonferroni adjustment was applied as appropriate.

RESULTS: Global cortical GM and WM volume was significantly reduced for each group comparison, except cortical GM volume of those with relapsing-remitting multiple sclerosis versus controls. Global and lobar cortical GM CBF and CBV were reduced in secondaryprogressive multiple sclerosis compared with other groups but not for relapsing-remitting multiple sclerosis versus controls. Global and lobar WM CBF and CBV were not significantly different across groups. The distribution of lobar cortical GM and WM volume reduction was disparate, except for the occipital lobes in patients with secondary-progressive multiple sclerosis versus those with relapsing-remitting multiple sclerosis. Moderate associations were identified between lobar cortical GM and lobar normal-appearing WM volume in controls and in the left temporal lobe in relapsing-remitting multiple sclerosis. No significant associations occurred between cortical GM and WM perfusion or volume. Strong correlations were observed between cortical-GM perfusion, normal appearing WM and lesional perfusion, with respect to each global and lobar region within $\mathrm{HC}$, and RRMS and SPMS patients $\left(R^{2} \leq 0.96, P<.006\right.$ and $\left.R^{2} \leq 0.738, P<.006\right)$.

CONCLUSIONS: The weak correlation between lobar WM and cortical GM volume loss and perfusion reduction suggests the independent pathophysiology of WM and cortical GM disease.

ABBREVIATIONS: $\mathrm{cGM}$ = cortical GM; EDSS = Expanded Disability Status Scale; $\mathrm{HC}=$ healthy control; NAWM = normal-appearing white matter; RRMS = relapsing-remitting multiple sclerosis; SPMS = secondary-progressive multiple sclerosis; T2h-1 = T2-hyperintense lesions

$S$ is a notable cause of neurologic and cognitive disability in young people. Pathologically, it is characterized by inflam- matory demyelination and, in chronic lesions, axonal loss. ${ }^{1}$ The cause of reduced cortical metabolism described in MS remains uncertain. ${ }^{2,3}$ While MS is typically regarded as a disease primarily affecting WM, cortical GM (cGM) is increasingly complicit in physical and cognitive disease progression. However, the relationships between WM and cGM disease progression remain controversial. Although some studies have suggested a relationship between normal-appearing WM (NAWM) atrophy and cGM damage ${ }^{4,5}$ others suggested that cGM disease progression is either independent from or only partly related to WM abnormali-

\footnotetext{
-- Indicates open access to non-subscribers at www.ajnr.org

三 Indicates article with supplemental on-line table.

$\checkmark$ Indicates article with supplemental on-line photos.

http://dx.doi.org/10.3174/ajnr.A5410
} 
ties. $^{6,7}$ Louapre et al, ${ }^{8}$ using DTI at 7T, found a lack of spatial specificity between NAWM tracts and the overlying cGM. Steenwijk et $\mathrm{al}^{9}$ reported a stronger relationship between cGM atrophy and WM tract pathology in patients with relapsing-remitting multiple sclerosis (RRMS) compared with those with secondaryprogressive multiple sclerosis (SPMS), concluding that the association between NAWM and cGM becomes increasingly independent with disease progression. The assertion that cGM and WM progression is either dependent or partly independent is supported by histopathologic and radiologic series demonstrating the role of meningeal mediated processes in both cortical and leucocortical lesions but not WM T2-hyperintense lesion (T2h-l) development. ${ }^{10,11}$

Although few studies ${ }^{4,5,9}$ have examined the regional relationship between cortical structure and WM disease, the association between regional WM volume and perfusion and cortical volume and perfusion has not been previously studied. CBF and CBV reduction have been previously shown either in the absence of, or adjusting for, intergroup structural differences, suggesting that cortical perfusion could serve as a surrogate of disease severity and tissue integrity under specific conditions. ${ }^{12-14}$ Aviv et al ${ }^{12}$ demonstrated focal cGM CBV reduction in cognitively impaired compared with preserved patients with SPMS after adjusting for global WM T2h-l volumes. Hojjat et $\mathrm{al}^{15}$ demonstrated significant CBF reduction in the absence of structural differences in impaired compared with cognitively preserved patients with RRMS. Last, Debernard et $\mathrm{al}^{14}$ found CBF reduction in the absence of cGM volume differences in unimpaired patients with RRMS compared with healthy controls (HCs) using pseudocontinuous arterial spin-labeled/labeling perfusion. While prior studies reported regional variation in $\mathrm{CBF}$ and $\mathrm{CBV}$, none examined the regional associations between lobar WM (normal-appearing and lesional) and cGM volume and perfusion reduction. Consistent with growing evidence for partly independent mechanisms of disease progression in WM and cGM, we hypothesized that an independent association would be found between lobar WM disease and cGM volume and perfusion.

\section{MATERIALS AND METHODS Study Participants}

Thirty-eight patients with MS (19 with SPMS and 19 with RRMS) from 2 tertiary referral MS clinics and 19 healthy, age-matched controls were prospectively recruited during a 1-year period. Exclusion criteria were a history of drug/alcohol abuse, relapse or steroid use in $<6$ months, pre-MS psychiatric history, head injuries involving loss of consciousness, cardiac disease, and MR imaging contraindications. Demographic data were obtained for each subject. This study was approved by both local ethics committees (Sunnybrook Health Sciences Centre and St. Michael's Hospital, Toronto), and written consent was obtained from each participant before study enrollment.

\section{Cognitive Testing}

All patients and HCs underwent MR imaging, neurologic examination, and Expanded Disability Status Scale (EDSS) assessment within 1 week. Patients were tested with the Minimal Assessment of Cognitive Function in Multiple Sclerosis battery comprising 7 tests covering 5 cognitive domains, including processing speed, memory, executive function, visuospatial perception, and verbal fluency. Only cognitively preserved patients were enrolled in the study, given the greater potential for confounding pathophysiologic factors, with greater disease progression characterized by cognitive impairment and the previously published association between cognitive impairment, disease progression, and hypoperfusion. ${ }^{9,15,16}$

\section{Image Acquisition}

All MR imaging data were acquired on a 3T MR imaging system (Achieva; Philips Healthcare, Best, the Netherlands) with an 8-channel phased array coil. The MR imaging sequences included: axial proton density $/ \mathrm{T} 2\left(\mathrm{TR} / \mathrm{TE} / \mathrm{flip}\right.$ angle $=2500 / 10.7 \mathrm{~ms} / 90^{\circ} ; \mathrm{FOV}=$ $230 \times 230 \mathrm{~mm}^{2}$; acquisition matrix $=256 \times 263$; section thickness $=3 \mathrm{~mm})$; axial T1-weighted TSE (TR/TE/flip angle $=9.5 /$ $2.3 \mathrm{~ms} / 12^{\circ} ; \mathrm{FOV}=240 \times 240 \mathrm{~mm}^{2} ;$ acquisition matrix $=256 \times$ 219; section thickness $=1.2 \mathrm{~mm}$ ); axial phase-sensitive inversion recovery $\left(\mathrm{TR} / \mathrm{TE}=3374 / 15 \mathrm{~ms}\right.$; FOV $=230 \times 230 \mathrm{~mm}^{2}$; acquisition matrix $=400 \times 255$; section thickness $=3 \mathrm{~mm}$ ); axial field-echo, echo-planar dynamic susceptibility contrast perfusion $\left(\mathrm{TR} / \mathrm{TE} /\right.$ flip angle $=1633 / 30 \mathrm{~ms} / 60^{\circ} ; \mathrm{FOV}=220 \times 220 \mathrm{~mm}^{2}$; acquisition matrix $=96 \times 93$; section thickness $=4 \mathrm{~mm}$; no gap; signal bandwidth $=1260 \mathrm{~Hz} /$ pixel; sections $=24$ ). During the perfusion scan, $10 \mathrm{~mL}$ of $1-\mathrm{mmol} / \mathrm{mL}$ concentrated gadobutrol (Gadovist; Bayer Schering Pharma, Berlin, Germany) was administered by a power injector at a rate of $5 \mathrm{~mL} / \mathrm{s}$, followed by a $25-\mathrm{mL}$ bolus of saline at $5 \mathrm{~mL} / \mathrm{s}$. Sixty images were acquired with the injection occurring at the fifth volume. A segmented inversion recovery Look-Locker EPI sequence was performed immediately before and after the axial DSC sequence (TR/TE/flip angle $=29$ / $14 \mathrm{~ms} / 20^{\circ}$; $\mathrm{TI}=15.8 \mathrm{~ms}$; FOV $=220 \times 220 \mathrm{~mm}^{2}$; acquisition matrix $=128 \times 126 ; 15 k$-space lines per acquisition; section thickness $=4 \mathrm{~mm}$; time points $=60$ ). A 3-second delay occurred following the last imaging time point to facilitate longitudinal magnetization recovery.

\section{Quantitative MR Perfusion}

Quantitative CBF (milliliter/100 gram/minute), quantitative CBV (milliliter/100 gram), and MTT (second) were obtained using bookend MR imaging perfusion as previously published. ${ }^{17}$ The technique uses pre- and postgadolinium bookend scans to calculate WM quantitative CBV without the need for an arterial input function while accounting for the effects of intravascular-toextravascular water exchange. The tissue concentration-time curve is calculated through arterial input function sampling, allowing relative $\mathrm{CBV}$ and relative $\mathrm{CBF}$ determination. The central volume principle was used to calculate MTT.

\section{Image Processing}

Structural T1- and proton-density/T2-weighted images were coregistered using linear registration (SPM8; http://www.fil. ion.ucl.ac.uk/spm/software/spm12). T2h-l and deep GM structures were segmented by a board-certified neuroradiologist $(>10$ years' experience) using the trace function in Analyze 8.0 (Mayo Clinic, Rochester, Minnesota). T1-weighted structural images were first segmented into GM and WM masks using the unified segmen- 
Table 1: Comparison of demographic and clinical data for HCs and subjects with RRMS and SPMS ${ }^{\mathrm{a}}$

\begin{tabular}{lcccccc}
\hline \multicolumn{1}{c}{ Parameter } & $\begin{array}{c}\text { HC } \\
(\boldsymbol{n}=\mathbf{1 9 )}\end{array}$ & $\begin{array}{c}\text { RRMS } \\
(\boldsymbol{n}=\mathbf{1 9})\end{array}$ & $\begin{array}{c}\text { SPMS } \\
(\boldsymbol{n}=\mathbf{2 0})\end{array}$ & $\begin{array}{c}\text { RRMS vs HC } \\
(\boldsymbol{P} \text { Value) }\end{array}$ & $\begin{array}{c}\text { SPMS vs HC } \\
(\boldsymbol{P} \text { Value) }\end{array}$ & $\begin{array}{c}\text { SPMS vs RRMS } \\
(\boldsymbol{P} \text { Value) }\end{array}$ \\
\hline Age (yr) & $49.0 \pm 7.1$ & $46.4 \pm 7.2$ & $55.2 \pm 6.5$ & .27 & $.0168^{\mathrm{b}}$ & $.0041^{\mathrm{b}}$ \\
Sex (F/M) & $14: 5$ & $15: 4$ & $11: 9$ & .70 & .23 & .12 \\
Education (yr) & $16.9 \pm 2.9$ & $16.1 \pm 1.3$ & $15.1 \pm 2.6$ & .22 & .051 & .15 \\
Disease duration (yr) & 0.00 & $11.8 \pm 5.4$ & $16.7 \pm 6.5$ & NA & NA & .0234 \\
EDSS (median) (IQR) & 0.00 & $1.5(1-2)$ & $6(6-6.5)$ & NA & NA & $.0006^{\mathrm{b}}$ \\
\hline
\end{tabular}

Note:-IQR indicates interquartile range; NA, not applicable.

${ }^{a}$ All values are mean (SD) except where indicated. Bonferroni corrected $P<.017$ is considered statistically significant.

b Significant.

tation model in SPM8 and checked for accuracy before creating subject-specific NAWM masks by subtracting T2h-1 from the automated WM segmentation. For cortical volumetric analysis, the International Consortium for Brain Mapping lobar templates (Laboratory of Neuroimaging, Keck School of Medicine, Los Angeles, California) and MRIcro Brodmann templates (Neuropsychology Laboratory, Columbia, South Carolina) were registered to Montreal Neurological Institute 152 space using the normalize function in SPM. Structural T1-weighted images and associated lesional ROIs and lobar templates were coregistered to the EPI DSC pregadolinium images with linear registration (FMRIB Linear Image Registration Tool, FLIRT; https:/fsl.fmrib.ox.ac.uk/fsl/fslwiki/FLIRT) and nonlinear intensity modulation and multiresolution, nonlinear registration with 4 subsampling levels (FMRIB Nonlinear Registration Tool, FNIRT; http://fsl.fmrib.ox.ac.uk/fsl/fslwiki/FNIRT). Global and lobar CGM and WM volumetric and perfusion metrics were then quantified separately for bilateral frontal, parietal, temporal, and occipital lobes, as previously described. ${ }^{18}$

\section{Statistical Analysis}

Demographic, clinical, volumetric, and perfusion data were summarized for HCs and patients with RRMS, and SPMS using the mean and SD for continuous variables and proportions for categoric variables. To compare RRMS versus HC, SPMS versus HC, and SPMS versus RRMS for demographic variables (ie, age, sex, educational years, disease duration, and EDSS), we used the univariate logistic regression model. Significant confounding factors were determined and used for perfusion data analysis. A Bonferroni-corrected $P$ value $<.017$ (.05/3) was considered statistically significant for controlling for multiple comparisons among the 3 groups. To compare HC, RRMS, and SPMS cohort differences for the imaging parameter covariates (ie, CBF, CBV, MTT, lobar GM, and WM volume), we used a generalized linear model with a logit link function after adjusting for confounding factors. The GENMOD procedure in Statistical Analysis Software (SAS, Version 9.4 for Windows; SAS Institute, Cary, North Carolina) was performed to fit the model, with a Bonferroni-adjusted $P$ value $<$ .017 considered statistically significant. Confounding factors of age, disease duration, and EDSS were assessed for multicollinearity by examining tolerance and variance inflation factors (1/tolerance) in a regression model using SPSS (IBM, Armonk, New York). A tolerance value of $<0.1$ and a variance inflation factor of $>10$ were regarded as indicating multicollinearity. Normality was determined with the Shapiro-Wilk test, and anomalous dependent variables were log-transformed to fit the data to a normal distribution. Natural log-transformation was applied as appropriate for normalizing the distributions. A general linear regres- sion was implemented to assess the association between GM and WM regional perfusion data, between GM and T2h-l regional perfusion data, between WM and T2h-l regional perfusion data, and between lobar GM and lobar WM volume data while considering confounding factors and expressed as $R^{2}$.

\section{RESULTS}

\section{Clinical Characteristics, Global Volumes, and Perfusion}

The MS subgroups did not significantly differ in sex, disease duration, and years of education, though the SPMS cohort was older $(P=.0041)$ and had higher EDSS $(P=.0006)$ scores than the RRMS cohort (Table 1). Patients with SPMS demonstrated a longer disease duration, but this did not reach statistical significance $(P=.02)$. The SPMS cohort had greater global atrophy in cGM and WM compared with the RRMS group and HC subjects ( $P=$ $.002, P=.0026$ and $P=.0049$, and $P=.0011$, respectively; Table $2)$. Those with RRMS exhibited lower global WM $(P=.0115)$ but not GM volume compared with HCs. GM and WM CBF and CBV were reduced and MTT was prolonged in subjects with SPMS compared with those with RRMS. GM CBF reduction and MTT prolongation were present in RRMS and SPMS compared with HCs. No significant WM CBF or CBV difference was observed for any RRMS/SPMS comparison with HCs. WM MTT was significantly prolonged for those with SPMS versus HCs. No significant T2h-l volume, CBF, or MTT differences were seen between patients with RRMS and those with SPMS, though patients with SPMS had higher T2h-l CBV than those with RRMS.

\section{Lobar Volumetric Group Comparisons}

Patients with SPMS had reduced cGM volumes in the temporal and occipital lobes and reduced WM volumes in the occipital lobe compared with those with RRMS (Table 3). Patients with SPMS also demonstrated reduced occipital lobe WM and temporal and occipital cGM compared with HCs. Frontal and parietal lobe WM volume reduction was observed for all comparisons, and patients with RRMS also demonstrated a reduced temporal WM volume compared with HCs. Overall, a weak association was present between lobar cGM and lobar NAWM volume (On-line Table and On-line Fig 1$)$ in patients with both RRMS $\left(R^{2} 0.14-0.65\right)$ and SPMS $\left(R^{2}=0.16-0.62\right)$, with no statistical significance achieved for any lobar region. Association was stronger in $\mathrm{HCs}\left(R^{2}=\right.$ $0.53-0.79)$.

\section{Lobar Perfusion Group Comparisons}

The distribution of significant lobar cortical perfusion differences between group comparisons is demonstrated in the Figure. Lobar cGM CBF reduction and MTT increase were present in all lobes in 
Table 2: Comparison of perfusion and volumetric data for CGM, NAWM, and T2h-l between HCs and subjects with RRMS and SPMS after adjusting for confounding factors ${ }^{\mathrm{a}}$

\begin{tabular}{|c|c|c|c|c|c|c|}
\hline \multirow[b]{2}{*}{ Parameter } & \multicolumn{3}{|c|}{ Median (Interquartiles) } & \multicolumn{3}{|c|}{$P$ Value } \\
\hline & $\mathrm{HC}(n=19)$ & RRMS $(n=19)$ & SPMS $(n=20)$ & RRMS vs HC & SPMS vs HC & SPMS vs RRMS \\
\hline \multicolumn{7}{|l|}{ cGM } \\
\hline Global volume & $890(869-914)$ & $864(827-895)$ & $673(646-697)$ & .10 & $.0026^{\mathrm{b}}$ & $.0020^{\mathrm{b}}$ \\
\hline CBF & $43.20(33.91-54.49)$ & $41.07(29.85-55.68)$ & $34.05(27.19-43.68)$ & $<.0001^{\mathrm{b}}$ & $<.0001^{\mathrm{b}}$ & $.0011^{\mathrm{b}}$ \\
\hline CBV & $2.64(2.02-3.29)$ & $2.53(1.94-3.37)$ & $2.40(1.92-3.07)$ & .078 & .046 & $.0062^{b}$ \\
\hline MTT & $3.77(3.20-4.31)$ & $3.90(3.32-4.41)$ & $4.30(3.66-4.99)$ & $.0001^{b}$ & $<.0001^{b}$ & $<.0001^{\mathrm{b}}$ \\
\hline \multicolumn{7}{|l|}{ NAWM } \\
\hline Global volume & $878(812-894)$ & $818(738-850)$ & 697 (669-729) & $.0115^{\mathrm{b}}$ & $.0011^{\mathrm{b}}$ & $.0049^{b}$ \\
\hline CBF & 23.49 (18.69-27.78) & $21.55(16.05-29.50)$ & 19.54 (16.74-24.92) & .68 & .58 & $.0080^{\mathrm{b}}$ \\
\hline CBV & $1.59(1.28-2.00)$ & $1.57(1.17-2.15)$ & $1.51(1.30-1.98)$ & .89 & .16 & $.0103^{\mathrm{b}}$ \\
\hline MTT & $4.51(4.09-5.10)$ & $4.62(4.08-5.20)$ & $4.86(4.28-5.41)$ & .25 & $.0005^{b}$ & $.0094^{\mathrm{b}}$ \\
\hline \multicolumn{7}{|l|}{ T2h-l } \\
\hline Global volume & 0.00 & $5.4(1.2-9.3)$ & $6.4(1.0-10.9)$ & NA & NA & .82 \\
\hline $\mathrm{CBF}$ & 0.00 & $12.77(8.23-17.24)$ & 11.15 (8.95-15.71) & NA & NA & .049 \\
\hline CBV & 0.00 & $0.99(0-1.32)$ & $1.08(0.83-1.47)$ & NA & NA & $.0026^{\mathrm{b}}$ \\
\hline MTT & 0.00 & $4.91(4.00-5.63)$ & $5.64(4.82-6.30)$ & NA & NA & .43 \\
\hline
\end{tabular}

Note:-NA, not applicable.

a Bonferroni-corrected $P<.017$ is considered statistically significant. Age was considered a confounding factor for comparing RRMS vs HC and SPMS vs HC; age, EDSS, and disease duration were considered confounding factors for comparing SPMS vs RRMS. All volumes are in cubic centimeters; CBF, milliliter/100 gram/meter; CBV, milliliter/100 gram; MTT, second.

Significant.

Table 3: Lobar GM and WM volume differences among HCs and subjects with RRMS and SPMS after adjusting for confounding factors ${ }^{a}$

\begin{tabular}{|c|c|c|c|c|c|c|}
\hline \multirow[b]{2}{*}{ Parameter } & \multicolumn{3}{|c|}{ Median (Interquartiles) } & \multicolumn{3}{|c|}{$P$ Value } \\
\hline & $\mathrm{HC}(n=19)$ & $\operatorname{RRMS}(n=19)$ & SPMS $(n=19)$ & $\begin{array}{c}\text { RRMS vs HC } \\
(n=19)\end{array}$ & $\begin{array}{c}\text { SPMS vs HC } \\
(n=19)\end{array}$ & $\begin{array}{c}\text { SPMS vs RRMS } \\
(n=19)\end{array}$ \\
\hline \multicolumn{7}{|l|}{ Gray matter } \\
\hline Frontal lobe & $200.040(190.352-167.944)$ & $199.248(192.480-207.896)$ & $200.352(193.500-207.540)$ & 69 & .60 & .22 \\
\hline Parietal lobe & $90.296(83.992-94.512)$ & $89.824(85.048-95.176)$ & $93.368(88.956-96.352)$ & .72 & .03 & .095 \\
\hline Temporal lobe & $122.740(110.640-130.560)$ & $121.336(114.672-128.544)$ & $106.892(99.432-112.928)$ & .86 & $.0044^{\mathrm{b}}$ & $.0073^{\mathrm{b}}$ \\
\hline Occipital lobe & $101.088(98.536-106.272)$ & $97.160(95.728-102.960)$ & $72.792(69.868-75.196)$ & .10 & $.0030^{\mathrm{b}}$ & $.0033^{b}$ \\
\hline \multicolumn{7}{|c|}{ White matter } \\
\hline Frontal lobe & 190.568 (170.368-201.528) & 170.316 (159.520-189.112) & $146.260(134.908-157.136)$ & $<.0001^{\mathrm{b}}$ & $.0006^{\mathrm{b}}$ & $<.0001^{\mathrm{b}}$ \\
\hline Parietal lobe & $101.200(94.536-109.152)$ & $93.272(84.944-97.208)$ & $81.952(77.736-87.532)$ & $.0001^{b}$ & $.0018^{\mathrm{b}}$ & $<.0001^{\mathrm{b}}$ \\
\hline Temporal lobe & $70.132(56.648-79.352)$ & $68.512(64.116-74.016)$ & 61.208 (51.576-71.720) & .026 & .98 & $.0026^{\mathrm{b}}$ \\
\hline Occipital lobe & $32.024(30.248-35.092)$ & $29.092(24.136-33.768)$ & $26.864(23.552-31.832)$ & .65 & $.0098^{\mathrm{b}}$ & $.0003^{b}$ \\
\hline
\end{tabular}

a Bonferroni-corrected $P<.017$ is considered statistically significant. Age was considered a confounding factor for comparing RRMS vs HC and SPMS vs HC; age, EDSS, and disease duration were considered confounding factors for comparing SPMS vs RRMS. All volumes are in cubic centimeters.

bignificant.

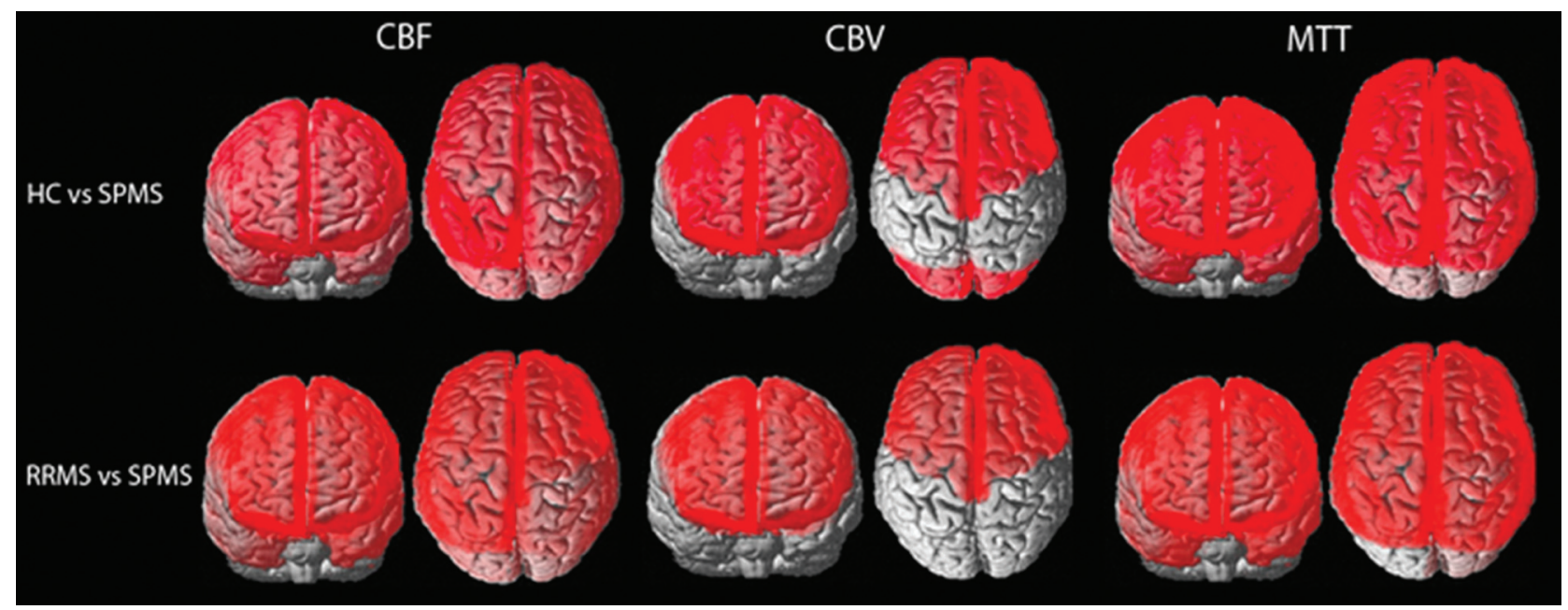

FIGURE. Whole-brain depiction of perfusion differences in cortical gray matter among HCs and patients with RRMS and SPMS. Units for CBF are milliliter/100 gram/minute; CBV, milliliter/100 gram; MTT, second. 
patients with SPMS compared with the other groups, except for MTT increase within the left occipital lobe for the SPMS versus RRMS comparison, which did not reach statistical significance. Lobar cGM CBV reduction was present in the bilateral frontal lobes of patients with SPMS compared with the other groups and in the bilateral occipital lobes of SPMS versus HCs. No significant lobar cGM CBF, CBV reduction, or MTT prolongation was found between subjects with RRMS and HCs. No significant lobar perfusion differences were observed between any group comparison for NAWM. A strong association was shown between cGM and NAWM global and lobar perfusion for all group comparisons and lobes $\left(R^{2}=0.77-0.98, P<.0001\right.$; On-line Table and On-line Fig 2). Overall, patients with SPMS demonstrated stronger associations between lobar T2h-l and cGM and NAWM perfusion compared with those with RRMS (On-line Table and On-line Figs 3 and 4; cGM CBF, $R^{2}=0.31-0.77$; NAWM CBF, $R^{2}=0.35-0.85$ versus cGM CBF, $R^{2}=0.07-0.61$; and NAWM CBF, $R^{2}=0.06-$ 0.69 , respectively).

\section{Associations between Perfusion and Volumetric Data}

No significant associations were found between perfusion and volumetric data in any regression analysis (ie, for cGM, T2h-l, or NAWM).

\section{DISCUSSION}

We demonstrated a weak association between lobar volumes of cGM and NAWM in patients with MS despite cGM and NAWM volume reduction with increasing disease severity. Similarly, although lobar NAWM and cGM perfusion were highly correlated, the distribution of lobar cGM perfusion reduction was distinct from that of underlying lobar NAWM perfusion, which showed no significant between-group differences. These results do not conflict with the notion that the pathophysiology of WM and cGM disease may occur independently and that the strength of association varies relative to the disease severity. The strong association between cGM and NAWM perfusion and the lack of association with volumetric measures suggest a potential role for perfusion as an independent surrogate of disease activity.

Weak associations between NAWM volume and GM volume and perfusion in the present study argue against a mechanism of secondary cGM anterograde or retrograde axonal degeneration and suggest independent pathophysiologic processes acting on WM and GM. This assertion is supported by various pathologic and imaging studies, ${ }^{8,19-21}$ which have shown that cGM lesions may develop before the appearance of WM plaques ${ }^{19}$ and arise independent of and are poorly correlated with T2h-l formation. $^{20,21}$ A number of pathologic and imaging articles have increasingly implicated an independent etiology for cGM lesion formation attributed to either the direct presence of meningeal-derived neurotoxic substances or secondary microglial activation mediated through meningeal/subpial inflammation and manifest as a gradient of demyelination centered on the subpial cortex. ${ }^{21,22}$

Numerous articles have examined the spatial relationship between lobar T2h-l and cGM integrity using quantitative and functional parameters other than perfusion. A recent correlative study of quantitative cortical $\mathrm{T}^{*}$ at $7 \mathrm{~T}$ - and $3 \mathrm{~T}$-derived surface- and tract-based analyses found a correlation between WM tract DTI and cGM integrity, though this was not spatially specific, reflecting a common sensitivity to MS pathologic changes. ${ }^{8}$ Steenwijk et $\mathrm{al}^{9}$ used DTI at 3T to investigate the association between regional GM atrophy and pathology in anatomically connected WM tracts in patients with RRMS, SPMS, and primary-progressive MS, demonstrating a relationship between NAWM tract fractional anisotropy and deep GM and cGM. The model of variance associated with cGM thickness was greatest in patients with RRMS but declined in those with SPMS and primary-progressive MS. A strong association between NAWM integrity and cGM thickness was found only in the mildly impaired group when patients were dichotomized by EDSS category 4. The authors concluded that NAWM integrity contributes to cGM atrophy only in early MS. Bodini et $\mathrm{al}^{4}$ used Tract-Based Spatial Statistics (TBSS; https://fsl.fmrib.ox.ac.uk/fsl/fslwiki/TBSS) to explore the relationship between cGM atrophy and fractional anisotropy in connected NAWM tracts in patients with primary-progressive MS and found that only 4/11 regions studied showed a quantitative association between reduced NAWM fractional anisotropy and GM atrophy.

Jehna et $\mathrm{al}^{5}$ found spatial interdependence among focal cortical volumes, lesion location, and probabilistic fiber pathways, suggesting that WM tracts and CGM volume are regionally dependent and injured due to similar disease processes, suggesting that lesional axonal transaction ${ }^{23}$ leads to Wallerian degeneration and retrograde GM atrophy. Their study was performed in "low disabled" individuals with significantly lower ages (29.5 years) and disease duration (7.3 years) compared with the present cohort. In contradistinction, we did not demonstrate a stronger association between NAWM/T2h-l and cGM volume or perfusion with earlier disease, likely explained by longer disease duration and older age in our RRMS group compared with the cohort of Jehna et al and the different functional techniques used. The near-universally stronger CGM and NAWM perfusion association and deteriorating perfusion metrics with disease progression also confirmed in prior studies ${ }^{12,14,16,22,24}$ suggest that perfusion is sensitive to a common pathophysiologic mechanism reflecting concomitant but not necessarily codependent cGM and WM pathology in MS. Findings are supported by a recently reported DTI study, ${ }^{8}$ suggesting that perfusion could serve as a useful surrogate of disease activity in addition to routine structural imaging.

Limitations of the study are the lobar rather than functional domain approach adopted to examine associations among NAWM, T2h-l, and cGM. This could result in functionally unrelated regions being included in the lobar cGM assessed. However, a lobar approach was previously used in a recent publication showing that the presence of juxtacortical T2h-l may affect the degree of lobar cortical thinning. ${ }^{25}$ Alternative approaches assessing the association between large-scale functional brain networks and cGM integrity may provide greater insight into the volumetric and functional spatial relationship and the effect on cognition. ${ }^{26}$ Greater insight into the association among NAWM, T2h-l, and cGM may be illustrated by a longitudinal rather than a crosssectional study design, therefore representing a limitation of the present study. Last, the small sample size is relatively modest, limiting generalizability to a broader population of patients with 
MS. Despite these sample size limitations, we could demonstrate important differences in associations between volumetric and perfusion variables.

\section{CONCLUSIONS}

The weak spatial association between WM disease and cGM atrophy does not conflict with the notion of an independent pathophysiology of WM and cGM disease. Perfusion reduction with disease severity, particularly in cGM, suggests that perfusion is sensitive to the pathophysiologic mechanism of MS disease severity and may be a useful surrogate of cortical disease progression.

Disclosures: Seyed-Parsa Hojjat—RELATED: Grant: Biogen fellowship funding award. Timothy J. Carroll—RELATED: Grant: National Institutes of Health (1R21EB017928-01A1). Charles G. Cantrell—RELATED: Grant: American Heart Association (14PRE20380310). Richard I. Aviv—RELATED: Grant: Canadian Institutes of Health Research (130366), Biogen fellowship funding award.

\section{REFERENCES}

1. Rashid W, Parkes LM, Ingle GT, et al. Abnormalities of cerebral perfusion in multiple sclerosis. J Neurol Neurosurg Psychiatry 2004; 75:1288-93 CrossRef Medline

2. De Keyser J, Steen C, Mostert JP, et al. Hypoperfusion of the cerebral white matter in multiple sclerosis: possible mechanisms and pathophysiological significance. J Cereb Blood Flow Metab 2008; 28:1645-51 CrossRef Medline

3. Sun X, Tanaka M, Kondo S, et al. Clinical significance of reduced cerebral metabolism in multiple sclerosis: a combined PET and MRI study. Ann Nucl Med 1998;12:89-94 CrossRef Medline

4. Bodini B, Khaleeli Z, Cercignani M, et al. Exploring the relationship between white matter and gray matter damage in early primary progressive multiple sclerosis: an in vivo study with TBSS and VBM. Hum Brain Map 2009;30:2852-61 CrossRef Medline

5. Jehna M, Langkammer C, Khalil M, et al. An exploratory study on the spatial relationship between regional cortical volume changes and white matter integrity in multiple sclerosis. Brain Connect 2013; 3:255-64 CrossRef Medline

6. Calabrese M, Gallo P. Magnetic resonance evidence of cortical onset of multiple sclerosis. Mult Scler 2009;15:933-41 CrossRef Medline

7. Fisher E, Lee JC, Nakamura K, et al. Gray matter atrophy in multiple sclerosis: a longitudinal study. Ann Neurol 2008;64:255-65 CrossRef Medline

8. Louapre C, Govindarajan ST, Gianni C, et al. Is the relationship between cortical and white matter pathologic changes in multiple sclerosis spatially specific? A multimodal 7-T and 3-T MR imaging study with surface and tract-based analysis. Radiology 2016;278: 524-35 CrossRef Medline

9. Steenwijk MD, Daams M, Pouwels PJ, et al. Unraveling the relationship between regional gray matter atrophy and pathology in connected white matter tracts in long-standing multiple sclerosis. Hum Brain Map 2015;36:1796-807 CrossRef Medline

10. Mainero C, Louapre C, Govindarajan ST, et al. A gradient in cortical pathology in multiple sclerosis by in vivo quantitative $7 \mathrm{~T}$ imaging. Brain 2015;138:932-45 CrossRef Medline
11. Klaver R, De Vries HE, Schenk GJ, et al. Grey matter damage in multiple sclerosis: a pathology perspective. Prion 2013;7:66-75 CrossRef Medline

12. Aviv RI, Francis PL, Tenenbein R, et al. Decreased frontal lobe gray matter perfusion in cognitively impaired patients with secondaryprogressive multiple sclerosis detected by the bookend technique. AJNR Am J Neuroradiol 2012;33:1779-85 CrossRef Medline

13. Peruzzo D, Castellaro M, Calabrese M, et al. Heterogeneity of cortical lesions in multiple sclerosis: an MRI perfusion study. J Cereb Blood Flow Metab 2013;33:457-63 CrossRef Medline

14. Debernard L, Melzer TR, Van Stockum S, et al. Reduced grey matter perfusion without volume loss in early relapsing-remitting multiple sclerosis. J Neurol Neurosurg Psychiatry 2014;85:544-51 CrossRef Medline

15. Hojjat SP, Cantrell CG, Vitorino R, et al. Regional reduction in cortical blood flow among cognitively impaired adults with relapsingremitting multiple sclerosis patients. Mult Scler 2016;11:1421-28 CrossRef Medline

16. Hojjat SP, Cantrell CG, Carroll TJ, et al. Perfusion reduction in the absence of structural differences in cognitively impaired versus unimpaired RRMS patients. Mult Scler 2016;22:1685-94 CrossRef Medline

17. Vakil P, Lee JJ, Mouannes-Srour JJ, et al. Cerebrovascular occlusive disease: quantitative cerebral blood flow using dynamic susceptibility contrast MR imaging correlates with quantitative $\mathrm{H} 2$ [150] PET. Radiology 2013;266:879-86 CrossRef Medline

18. Shah MK, Shin W, Parikh VS, et al. Quantitative MR perfusion imaging: preliminary results in stroke. J Magn Reson Imaging 2010; 32:796-802 CrossRef Medline

19. Popescu BF, Lucchinetti CF. Meningeal and cortical grey matter pathology in multiple sclerosis. BMC Neurol 2012;12:111 CrossRef Medline

20. Bö L, Geurts JJ, van der Valk P, et al. Lack of correlation between cortical demyelination and white matter pathologic changes in multiple sclerosis. Arch Neurol 2007;64:76-80 CrossRef Medline

21. Sethi V, Yousry T, Muhlert N, et al. A longitudinal study of cortical grey matter lesion subtypes in relapse-onset multiple sclerosis. J Neurol Neurosurg Psychiatry 2016;87:750-53 CrossRef Medline

22. Francis PL, Jakubovic R, O'Connor P, et al. Robust perfusion deficits in cognitively impaired patients with secondary-progressive multiple sclerosis. AJNR Am J Neuroradiol 2013;34: 62-67 CrossRef Medline

23. Trapp BD, Peterson J, Ransohoff RM, et al. Axonal transection in the lesions of multiple sclerosis. N Engl J Med 1998;338:278-85 CrossRef Medline

24. Francis PL, Chia TL, Jakubovic R, et al. Extensive white matter dysfunction in cognitively impaired patients with secondary-progressive multiple sclerosis. AJNR Am J Neuroradiol 2014;35:1910-15 CrossRef Medline

25. Pareto D, Sastre-Garriga J, Auger C, et al. Juxtacortical lesions and cortical thinning in multiple sclerosis. AJNR Am J Neuroradiol 2015; 36:2270-76 CrossRef Medline

26. Gläscher J, Rudrauf D, Colom R, et al. Distributed neural system for general intelligence revealed by lesion mapping. Proc Natl Acad Sci U S A 2010;107:4705-09 CrossRef Medline 